\title{
Skin cancer excision performance in Scottish primary and secondary care:
}

\author{
a retrospective analysis
}

\begin{abstract}
Background

In contrast with most published evidence, studies from north-east Scotland suggest that GPs may be as good at treating skin cancers in primary care as secondary care specialists.
\end{abstract}

\section{Aim}

To compare the quality of skin cancer excisions of GPs and secondary care skin specialists in east and south-east Scotland.

\section{Design and setting}

A retrospective analysis of reports from GPs in Lothian, Fife, and Tayside regions.

\section{Method}

Skin cancer histopathology reports from GPs in Lothian, Fife, and Tayside regions in 2010 were compared with reports from skin specialists in November 2010. The histopathology reports were rated for completeness and adequacy of excision.

\section{Results}

A total of 944 histopathology reports were analysed. In 1 year, GPs biopsied or excised 380 skin cancers. In 1 month, dermatologists biopsied or excised 385 skin cancers, and plastic surgeons 179 skin cancers. 'High risk' basal cell carcinomas (BCC) comprised $63.0 \%$ of BCC excised by GPs. For all skin cancer types, GPs excised smaller lesions, and had a lower rate of complete excisions compared with skin specialists. A statistical difference was demonstrated for BCC excisions only.

\section{Conclusion}

GPs in east and south-east Scotland excise a number of skin cancers including malignant melanoma (MM), squamous cell carcinoma (SCC) and high-risk BCC. Despite removing smaller lesions, less commonly on difficult surgical sites of the head and neck, GP excision rates are lower for all skin cancers, and statistically inferior for BCC, compared with secondary care, supporting the development of guidelines in Scotland similar to those in other UK regions. Poorer GP excision rates may have serious consequences for patients with highrisk lesions.

\section{Keywords}

basal cell carcinoma; melanoma; primary health care; secondary care; skin surgery; squamous cell carcinoma.

\section{INTRODUCTION}

Skin cancer is the most common type of malignancy in the UK. ${ }^{1}$ In 2010, there were approximately 99549 cases of non-melanoma skin cancers (NMSC), predominantly basal cell carcinoma (BCC) and squamous cell carcinoma (SCC), and 12818 new cases of melanoma (MM) registered. The incidence of all three continues to rise. ${ }^{1,2}$ The total cost of skin cancer to the NHS is projected to be over £180 million in 2020, constituting a substantial burden to the British health services. $^{3}$

There are currently no national guidelines in Scotland regarding primary care management of BCC and SCC, although the Scottish Intercollegiate Guidelines Network (SIGN) has recently proposed a guideline on management of SCC, which is due to be published in 2014. SIGN number 72 on melanoma states:

GPs should refer urgently all patients in whom melanoma is a strong possibility rather than carry out a biopsy in primary care.' ${ }^{4}$

In contrast, the National Institute for Health and Care Excellence (NICE) guidance for England recommends that all skin lesions suspicious of skin cancers, except low-risk BCC, should be referred urgently to secondary care and not be treated in the community. 5,6 In Scotland, like the rest of the UK, patients suspected to have skin cancer present initially to their GPs, who act as gatekeepers to secondary care services. Thus most UK GPs are skilled in efficient triage of large numbers of benign skin lesions and referral of potential malignancies. However, there are concerns of insufficient GP training in the diagnosis and especially treatment of skin cancer, with most studies reporting higher rates of incomplete excision for NMSC in primary care. ${ }^{7.8}$ Studies from north-east Scotland found inferior treatment of BCC in primary care, but that the GPs excision rates for SCC were as good as secondary care skin specialists, and that long-term outcomes for patients with MM were no different if the initial diagnostic excision was undertaken in primary or secondary care. ${ }^{9-11}$ These results were based on historical data, pre-dating the NICE and SIGN guidelines, and before the introduction of one-stop dermatology skin cancer clinics, which allow rapid specialist assessment, treatment, and multidisciplinary team management. To assess current practice and outcomes, a retrospective study was undertaken looking at recent performances of GPs and skin specialists in excising the three common skin cancers in east and south-east Scotland.
WY Haw, MBChB, foundation doctor, University of Edinburgh Medical School, Edinburgh, UK.

P Rakvit, MD, dermatology registrar;

AG Affleck, BSc, MBChB, consultant dermatologist Department of Dermatology, Ninewells Hospital, Dundee, UK. SJ Fraser, MBChB, consultant dermatologist, Department of Dermatology, Queen Margaret Hospital, Dunfermline, UK. SA Holme, $\mathrm{BSc}, \mathrm{MBChB}, \mathrm{MD}$, consultant dermatologist, Department of Dermatology, Royal Infirmary of Edinburgh, Edinburgh, UK.

\section{Address for correspondence}

Dr SA Holme, Royal Infirmary of Edinburgh,
Lauriston Building, Lauriston Place, Edinburgh, EH3 9HA, UK.

E-mail: alex.holmeanhs.net

Submitted: 15 January 2014; Editor's response: 5 February 2014; Final acceptance:

11 April 2014.

\section{OBritish Journal of General Practice}

This is the full-length article (published online 28 July 2014) of an abridged version published in print. Cite this article as: $\mathbf{B r} \mathbf{J}$ Gen Pract 2014; DOI: 10.3399/bjgp14X680929 


\section{How this fits in}

NICE recommends that all skin cancer, except low-risk BCC, should be referred to secondary care, but there are no similar guidelines for $\mathrm{BCC}$ and $\mathrm{SCC}$ in Scotland. SIGN has previously published MM guidance and will publish SCC guidance later in 2014. Research from an adjacent Scottish Health Board region, using a historic dataset that pre-dates national skin cancer management guidelines and dermatology skin cancer service reconfiguration, suggests poorer performance for primary care BCC surgery but not for SCC surgery. Using recent data across three Scottish Health Boards, an evidence base is provided to support the production of guidance and education for Scottish GPs assessing and referring skin cancers or performing cutaneous surgery. The demonstration of poorer primary care excision of patients' BCC compared with secondary care, which may be of clinical consequence for high-risk lesions, supports development of guidelines in Scotland for BCC similar to those in other UK regions.

\section{METHOD}

Histopathology reports were reviewed for all three common skin cancer diagnoses (BCC, SCC, and MM) undertaken in three east and south-east Scotland health board regions (Lothian, Fife, and Tayside) covering a population of 1.6 million. The pathology reports of GPs' skin surgery between 1 January and 31 December 2010 were compared with those of skin specialists in the month of November 2010. These two time periods were used to provide comparable numbers of primary care and secondary care cases, and the month of November for skin specialists was chosen to correspond with the most recent 5-yearly detailed assessments of south-east

Table 1. Comparison of surgery type and proportion between GPs (total number 1 year 2010), dermatologists, and plastic surgeons (total number November 2010)

\begin{tabular}{llccc} 
Skin cancer type & Surgery type & GPs & Dermatologists & Plastic surgeons \\
\hline BCC & Excision, \% $(n)$ & $78.2(208)$ & $53.7(160)$ & $93.7(134)$ \\
& Diagnostic, \% $(n)$ & $21.8(58)$ & $46.3(138)$ & $6.3(9)$ \\
\hline SCC & Excision, \% $(n)$ & $69.3(61)$ & $37.3(25)$ & $96.6(28)$ \\
& Diagnostic, \% $(n)$ & $30.7(27)$ & $62.7(42)$ & $3.4(1)$ \\
\hline MM & Excision, \% $(n)$ & $100.0(26)$ & $90.0(18)$ & $100.0(7)$ \\
& Diagnostic, \% $(n)$ & $0.0(0)$ & $10.0(2)$ & $0.0(0)$ \\
\hline & Unclear, $(n)$ & $(12)$ & $(9)$ & $(2)$ \\
\hline
\end{tabular}

$B C C=$ basal cell carcinoma. $M M=$ malignant melanoma. $S C C=$ squamous cell carcinoma
Scotland dermatology outpatient activity. ${ }^{12}$

Data obtained included age, sex, diagnosis, maximum diameter of lesion, site of specimen, histological clearance, excision margin, and the specialty of the medical practitioner performing the surgery. Non-invasive premalignant lesions were excluded (for example melanoma-in-situ, SCC-in-situl. Excision was interpreted as removal of a skin lesion with the intention to treat. Incision, punch, shave, or curettage, were classified as diagnostic procedures. Completeness of excision was recorded as reported by the pathologist. Adequacy of excision was taken to be clearance of radial and deep margins greater than $0.5 \mathrm{~mm}$. The specialty of medical practitioner (GP, dermatologist, or plastic surgeon) was determined by the specialty recorded on the pathology database or report. Skin cancers excised by other specialties were excluded from the study. High-risk BCC were defined, as in the NICE guideline, as recurrent BCC, infiltrative BCC, or BCC located on the head and neck regions.

Analyses were conducted using SPSS (version 18). Mean age ( \pm standard deviation [SD]) of patients and the types of skin cancers seen in primary (GPs) and secondary care (dermatologists and plastic surgeons) were compared using analysis of variance. The completeness of excision and adequacy of margins among GPs, dermatologists, and plastic surgeons were compared using the $\chi^{2}$ test.

\section{RESULTS}

A total of 944 histopathology reports (707 BCC, 184 SCC, and 53 MM) were assessed. In the whole year of 2010, GPs biopsied or excised 380 skin cancers (266 BCC, 88 SCC, 26 MM); compared with 385 by dermatologists (298 BCC, 67 SCC, 20 MM) and 179 (143 BCC, 29 SCC, 7 MM) by plastic surgeons in the month of November 2010. An additional 24 BCC, 17 SCC, and 0 $\mathrm{MM}$ were excised by other secondary care specialties in November 2010 (maxillofacial surgery; ophthalmology; breast surgery; ear, nose, and throat; oncology; and general surgeryl, and were not included in the detailed analysis

Skin cancers were more common in males than females ( $58 \%$ versus $42 \%$ ), and the proportions of sexes were similar in primary and secondary care. There were significant differences between the proportions of BCC, SCC, and MM seen in primary or secondary care $(70.0 \% \mathrm{BCC}$, $23.2 \%$ SCC, $6.8 \% \mathrm{MM}$ in primary care versus $78.2 \% \mathrm{BCC}, 17.0 \%$ SCC, $4.8 \% \mathrm{MM}$ in secondary care; $P=0.017)$. The mean 
Table 2. Site of surgery according to type of skin cancer and specialty

\begin{tabular}{llccc} 
Skin cancer type & Site & GPs, $\%(\boldsymbol{n})$ & Dermatologists, $\%(\boldsymbol{n})$ & Plastic surgeons, $\%(\boldsymbol{n})$ \\
\hline BCC & Head and neck & $53.0(141)$ & $71.8(214)$ & $83.9(120)$ \\
& Anterior trunk & $18.0(48)$ & $10.1(30)$ & $4.9(7)$ \\
& Posterior trunk & $15.0(40)$ & $7.4(22)$ & $2.8(4)$ \\
& Lower limb & $8.3(22)$ & $5.4(16)$ & $3.5(5)$ \\
& Upper limb & $5.6(15)$ & $5.4(16)$ & $4.9(7)$ \\
\hline SCC & Head and neck & $47.7(42)$ & $65.7(44)$ & $79.3(23)$ \\
& Anterior trunk & $3.4(3)$ & $1.5(1)$ & $3.4(1)$ \\
& Posterior trunk & $5.7(5)$ & $6.0(4)$ & $0.0(0)$ \\
& Lower limb & $18.2(16)$ & $16.4(11)$ & $10.3(3)$ \\
& Upper limb & $25.0(22)$ & $10.4(7)$ & $6.9(2)$ \\
\hline MM & Head and neck & $0.0(0)$ & $30.0(6)$ & $57.1(4)$ \\
& Anterior trunk & $23.1(6)$ & $20.0(4)$ & $14.3(1)$ \\
& Posterior trunk & $15.4(4)$ & $10.0(2)$ & $0.0(0)$ \\
& Lower limb & $38.5(10)$ & $20.0(4)$ & $0.0(0)$ \\
& Upper limb & $23.1(6)$ & $20.0(4)$ & $28.6(2)$ \\
\hline
\end{tabular}

$B C C=$ basal cell carcinoma. $M M=$ malignant melanoma. $S C C=$ squamous cell carcinoma
$20 \%$ for dermatologists, and $0 \%$ for plastic surgeons) however, this trend was reversed with more MM excisions from the face and neck regions in secondary care than by GPs $10 \%$ for GPs, 30\% for dermatologists, and $57.1 \%$ for plastic surgeons).

Documentation of lesion diameter was available for $64 \%$ of excisions $166.3 \%$ BCC, $51.6 \%$ SCC, and $75.5 \%$ MM). Table 3 demonstrates that average diameter of GP excised lesions was smaller for each skin cancer type than dermatology and plastic surgery, and this was significant for all types except high-risk BCC.

Table 4 illustrates when excision of lesion was intended, that GPs completely excised significantly fewer BCC and with less adequate margins than dermatologists or plastic surgeons. High-risk BCC accounted for $63.0 \%$ (131) of GP' BCC excisions, $76.9 \%$ (123) of dermatologists', and $85.1 \%$ (114) of plastic surgeons', and again there was a significantly poorer excision rate and adequacy of excision of high-risk BCC for GPs compared with secondary care. Comparing skin specialists, dermatologists' apparent higher rate of high-risk BCC excision and higher rate with margin greater than $0.5 \mathrm{~mm}$ compared with plastic surgeons was not statistically significant ( $P=0.98$ and $P=0.72$, respectively). For SCC, fewer GP excisions were complete, with fewer excised with an adequate margin compared with secondary care, but this was not significant. Dermatologists had a higher rate of complete SCC excision, and with adequate margin, compared with plastic surgeons, but again this was not statistically significant $(P=0.061$ and $P=0.935$, respectively). GPs completely excised fewer MM compared with secondary care, but this was not statistically significant.

\section{DISCUSSION}

\section{Summary}

The incidence of BCC, SCC, and MM, the most common forms of skin cancer in the UK, continues to increase, and diagnosis and optimal treatment can be challenging. Appropriate suspicion of skin cancer by GPs and referral to appropriate secondary services facilitate specialist diagnostic confirmation, earlytreatment, andintegration into multidisciplinary management networks. It has been shown previously that GPs' clinical diagnostic concordance with dermatologists for common inflammatory dermatoses is good lacne 94\%; psoriasis $89 \%$; and atopic dermatitis $77 \%$ ), but their diagnostic concordance for the common skin malignancies is poorer (BCC $43 \%$; SCC 


\begin{tabular}{|c|c|c|c|}
\hline Skin cancer type & Specialties & $\%$ Incomplete $(n)$ & $\%$ Complete excision $(n)$ \\
\hline Total skin cancers & $\begin{array}{l}\text { GPs } \\
\text { Dermatologists } \\
\text { Plastic surgeons } \\
\text { GP versus secondary care }\end{array}$ & $\begin{array}{c}23.1(68) \\
5.9(12) \\
10.7(18)\end{array}$ & $\begin{array}{c}76.9(227) \\
94.1(191) \\
89.3(151) \\
P<0.001 \\
\text { (adequate } P<0.001)\end{array}$ \\
\hline BCC & $\begin{array}{l}\text { GPs } \\
\text { Dermatologists } \\
\text { Plastic surgeons } \\
\text { GP versus secondary care }\end{array}$ & $\begin{array}{c}20.2(42) \\
5.6(9) \\
8.2(11)\end{array}$ & $\begin{array}{c}79.8(166) \\
94.4(151) \\
91.8(123) \\
P<0.001 \\
\text { ladequate } P<0.001)\end{array}$ \\
\hline High-risk BCC & $\begin{array}{l}\text { GPs } \\
\text { Dermatologists } \\
\text { Plastic surgeons } \\
\text { GP versus secondary care }\end{array}$ & $\begin{array}{c}22.1(29) \\
4.1(5) \\
9.6(11)\end{array}$ & $\begin{array}{c}77.9(102) \\
95.9(118) \\
90.4(103) \\
P<0.001 \\
\text { (adequate } P=0.005)\end{array}$ \\
\hline SCC & $\begin{array}{l}\text { GPs } \\
\text { Dermatologists } \\
\text { Plastic surgeons } \\
\text { GP versus secondary care }\end{array}$ & $\begin{array}{c}27.9(17) \\
4.0(1) \\
21.4(6)\end{array}$ & $\begin{array}{c}72.1(44) \\
96.0(24) \\
78.6(22) \\
P=0.055 \\
\text { ladequate } P=0.69)\end{array}$ \\
\hline MM & $\begin{array}{l}\text { GPs } \\
\text { Dermatologists } \\
\text { Plastic surgeons } \\
\text { GP versus secondary care }\end{array}$ & $\begin{array}{l}34.6(9) \\
11.1(2) \\
14.3(1)\end{array}$ & $\begin{array}{c}65.4(17) \\
88.9(16) \\
85.7(6) \\
P=0.057\end{array}$ \\
\hline
\end{tabular}

$B C C=$ basal cell carcinoma. $M M=$ malignant melanoma. $S C C=$ squamous cell carcinoma.
30\%; and MM 14\%).12 The key findings from this study are, in the treatment of common skin cancers in primary care in east and south-east Scotland, that smaller lesions are excised in primary care, that these are less commonly in the head and neck regions, and that excision rates are inferior in terms of complete excision and adequate excision margins compared with secondary care dermatologists and plastic surgeons. The findings of poorer recognition of skin malignancy by GPs and poorer quality of skin cancer surgery in primary care compared with secondary care have been reported in other UK studies. ${ }^{8,13-15}$ Additional concerns around primary care skin cancer treatment stem from fewer primary care MM excision patients receiving specialist management, and GPs reportedly making less use of pathology services than secondary care doctors when conducting minor surgery. 14,15 Internationally, similar trends are reported, although differences between healthcare models, dermatology, and skin surgery training influence outcomes and make translation of findings to the UK less valid. Failure to accurately diagnose, refer a patient urgently, or incompletely excise a lesion can result in increased morbidity, mortality, and patient distress. ${ }^{16}$

\section{Strengths and limitations}

This retrospective observational study has its strengths and limitations. Using a large reference population, and a recent dataset, all samples that were positive for skin cancers submitted by GPs over a 1-year period were studied, and this sample is comparable with the number of skin cancers treated by skin specialists in secondary care in 1 month, providing a large sample and meaningful comparison between the different specialties. A whole year of secondary care data would have provided a larger dataset, allowing meaningful examination of the other secondary care specialties, and perhaps permitting statistical significance in the SCC and MM primary-secondary care analyses. Using a pathology data source, it was not possible to determine the grade of the operator undertaking the surgery within each specialty nor to prospectively confirm that all treated patients with skin cancer have been captured llocally, all secondary care skin samples, whether suspected malignancy or not, are sent to histopathology, but primary care practice is not known). The study design did not allow capture of patient journey times to complete skin cancer treatment or assess patient comorbidities or satisfaction, which may be factors influencing GPs' decisions about site of surgery. Counterintuitively, this study showed that dermatologists appear to have a superior rate of complete excision of skin cancer compared with plastic surgeons, but that their overall surgical margins tend to be narrower. It seems unlikely that dermatologists are intrinsically superior surgeons to plastic surgeons, so the explanation instead may reflect larger, more complex cases being referred to plastic surgeons for excision, seniority of operating staff, or planned surgical margins, variables not assessed in the study. The smaller number of MM makes meaningful interpretation between dermatologists and plastic surgeons impossible.

Across all three tumour types, GPs proportionally excised fewer head and neck lesions than dermatologists and plastic surgeons. The reason for this is not clear, but may relate to the more complex anatomy, concerns about cosmesis, or appropriate wound closure when recommended margins are used. Of concern, this study showed that GPs tended to excise MM in younger patients, and refer older patients with MM to secondary care, yet GPs' excision rates for MM were lower compared with those of skin specialists. Thus, for the most dangerous of the three 
tumour types, young, economically active individuals are being put at excess risk of morbidity and mortality.

Although some parts of rural Scotland make access to treatment challenging, the authors believe that patient care should not be compromised by location. GPs with a specific interest and training in skin cancer management can offer optimum treatment in primary care through close links with skin cancer multidisciplinary teams and audit of their practice to demonstrate acceptable results. However, in the current absence of significant investment in primary care dermatological diagnostic skills and skin surgery training, and with UK health policy seeking to reduce delays to definitive cancer treatment, guidelines for primary care triage and referral of suspicious lesions for secondary care management appear to offer patients the best quality, and may be more cost-effective. ${ }^{4.14}$ Given the rising incidence of skin cancer, it is not clear whether this approach is sustainable. Policymakers may wish to consider this area of service provision, particularly the proportion of low-risk BCC currently managed in Scottish secondary care, which may be more suitable for treatment closer to the patient's home.

\section{Comparison with existing literature}

The main exceptions to the body of evidence supporting secondary care excision of all but low-risk BCC have come from a group in the adjacent Grampian health board region in north-east Scotland. They suggested that GPs excised SCC adequately compared with hospital skin specialists, and that the incomplete excision rate of GPs was comparable with secondary care incomplete excision rates. ${ }^{10}$ Some feel that these are anomalous results related to their inclusion of secondary care diagnostic biopsies in the incompletely excised categories, or their use of a historic dataset of results between 1991 and 2007, pre-dating the implementation of referral guidelines, dermatology onestop skin tumour clinics, and tracking of skin cancer referrals to ensure definitive treatment within specific time limits. ${ }^{17,18}$ To illustrate differences between their dataset compared with current practice illustrated in the present study, only $15.2 \%$ of $\mathrm{MM}$ excisions were undertaken by dermatology, and general surgeons undertook 21.8\% la specialty whose involvement in skin cancer work today would be regarded as unusual and who excised only one lesion in the current study sample).

\section{Implications for research and practice}

In conclusion, this study has demonstrated, using a recent cohort of patients with skin cancer, that Scottish GPs excise considerable numbers of MM, SCC, and high-risk BCC. GP excision rates were inferior to skin specialists, and for high-risk lesions this may have serious consequences. Not only do secondary care skin specialists appear to offer higher-quality excisions, but patients are also integrated into multidisciplinary team management, providing evidencebased care including follow-up, specialist nursing support, and patient information. NICE guidelines in England recommend that low-risk BCC can be managed by GPs and high-risk BCC, SCC, and MM should be referred to skin specialists. ${ }^{6}$ In Scotland, SIGN guideline number 72 advises secondary care management of cutaneous melanoma but national guidance for BCC and SCC is currently lacking. ${ }^{4}$ An evidence base has been provided to support the production of guidance and education for Scottish GPs assessing and referring skin cancers or performing cutaneous surgery. Assessment of larger populations with site, age, and tumour size matching may elucidate whether there are statistically significant differences between SCC and MM excision rates.

\section{Funding}

None.

\section{Ethical approval}

Ethical approval was not required.

\section{Provenance}

Freely submitted; externally peer reviewed.

\section{Competing interests}

The authors have declared no competing interests.

\section{Discuss this article}

Contribute and read comments about this article: www.bjgp.org/letters 


\section{REFERENCES}

1. Cancer Research UK. UK Cancer Incidence (2010) by Country Summary. CRUK, 2013 .http://publications.cancerresearchuk.org/downloads/Product/CS_DT_ INCCOUNTRIES.pdf (accessed 14 Jul 2014).

2. Information and Statistics Division. Cancer in Scotland. 2013. http://unw. isdscotland.org/Health-Topics/Cancer/Cancer-Statistics/Cancer_in_Scotland_ summary_m.pdf laccessed 10 Jun 2014)

3. Vallejo-Torres L, Morris S, Kinge JM, et al. Measuring current and future cost of skin cancer in England. J Public Health (Oxf) 2014; 36(1): 140-148.

4. Scottish Intercollegiate Guidelines Network. Cutaneous melanoma - a national clinical guideline. SIGN guideline number 72. Edinburgh: SIGN, 2003.

5. National Institute for Health and Clinical Excellence. Improving outcomes for people with skin tumours including melanoma. The manual. London: NICE, 2006. http://wnw.nice.org.uk/guidance/csgstim/resources/improving-outcomesfor-people-with-skin-tumours-including-melanoma-the-manual-2006guidance2 (accessed 14 Jul 2014).

6. National Institute for Health and Care Excellence. Improving outcomes for people with skin tumours including melanoma (update): the management of low risk basal cell carcinomas in the community (2010 partial guidance update). London: NICE, 2010.

7. Chen SC, Pennie ML, Kolm P, et al. Diagnosing and managing cutaneous pigmented lesions: primary care physicians versus dermatologists. J Gen Int Med 2006; 21(7): 678-682.

8. Khorshid SM, Pinney E, Bishop JA. Melanoma excision by general practitioners in north-east Thames region, England. Br J Dermatol 1998; 138(3): 412-417.
9. Murchie P, Sinclair E, Lee AJ. Primary excision of cutaneous melanoma: does the location of excision matter. Br J Gen Pract 2011; 61(583): 131-134.

10. Delaney EK, Duckworth L, Thompson WD, et al. Excising squamous cell carcinomas: comparing the performance of GPs, hospital skin specialists and other hospital specialists. Fam Practice 2012; 29(5): 541-546.

11. Murchie P, Delaney EK, Thompson WD, Lee AJ. Excising basal cell carcinomas: comparing the performance of general practitioners, hospital skin specialists and other hospital specialists. Clin Exp Dermatol 2008; 33(5): 565-571.

12. Holme SA, Scott-Lang VE, Ooi ET, et al. The south-east Scotland dermatology workload study: 30 years' analysis. Br J Dermatol 2012; 167(1): 123-130.

13. Pockney P, Primrose J, George S, et al. Recognition of skin malignancy by general practitioners: observational study using data from a population-based randomised controlled trial. Br J Cancer 2009; 100(1): 24-27.

14. George S, Pockney P. Primrose J, et al. A prospective randomised comparison of minor surgery in primary and secondary care. The MiSTIC trial. Health Tech Assess (Winchester, England) 2008; 12(23): iii-iv, ix-38.

15. Neal RD, Cannings-John R, Hood K, et al. Excision of malignant melanomas in North Wales: effect of location and surgeon on time to diagnosis and quality of excision. Fam Pract 2008; 25(4): 221-227.

16. McKenna DB, Marioni JC, Lee RJ, et al. A comparison of dermatologists', surgeons' and general practitioners' surgical management of cutaneous melanoma. Br J Dermatol 2004; 151(3): 636-644.

17. Hayes S, Keohane S. Primary excision of cutaneous melanoma. Br J Gen Pract 2011; 61(585): 290-291.

18. Muller F, Meredith F, Ormerod AD. Primary excision of cutaneous melanoma. $\mathrm{Br}$ $J$ Gen Pract 2011; 61(585): 291-292. 